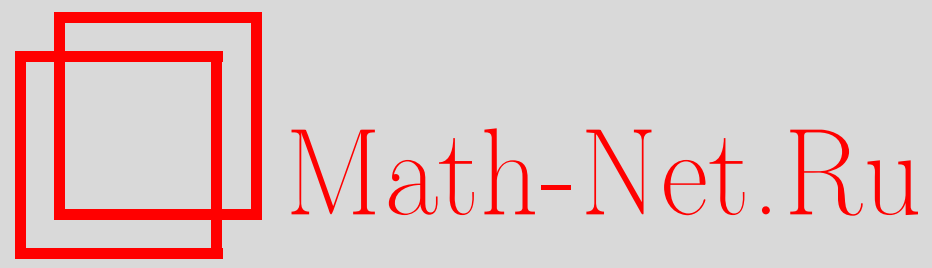

А. В. Забарило, А. Г. Завадский, Однопараметрические оснащенные частично упорядоченные множества и их представления, Функи. анализ и его прил., 2000, том 34, выпуск 2, 72-75

DOI: https://doi.org/10.4213/faa299

Использование Общероссийского математического портала MathNet.Ru подразумевает, что вы прочитали и согласны с пользовательским соглашением

http://www . mathnet.ru/rus/agreement

Параметры загрузки:

IP : 54.209 .52 .79

26 апреля 2023 г., 04:00:42

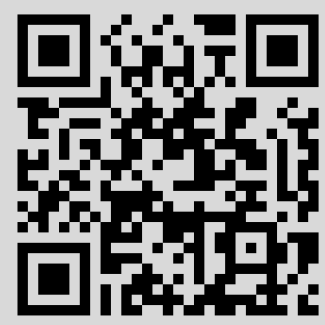


теорема Гиса в действительности является теоремой о четырех вершинах для временеподобных замкнутых кривых на $\Sigma \subset \mathbb{R} P^{1} \times \mathbb{R} P^{1}$ с лоренцевой метрикой постоянной кривизны (4).

Мы благодарны Л. Гийо за неоценимую помощь на начальной стадии работы, а также С. Лаззарини за полезные замечания.

\title{
ЛИТЕРАТУРА
}

1. Дубровин Б. А., Новиков С. П., Фоменко А. Т. Современная геометрия: Методы и приложения. Наука, 1979. 2. Duval C., Guieu L. J. Geom. Phys., 33, 103-127 (2000). 3. Ghys E. Cercles osculateurs et géométrie lorentzienne. Colloquium talk, Journée inaugurale du CMI, Marseille (February 1995). 4. Hubbard J. In: Riemann surfaces and related topics. Proc. of the Stony Brook Conference 1978 (I. Kra and B. Maskit, eds.). Ann. of Math. Stud., 97, Princeton Univ. Press, Princeton, N.J., 1981, pp. 257-275. 5. Kostant B., Sternberg S. In: Quantum theories and geometry (M. Cahen and M. Flato, eds.). Kluwer Acad. Publ., Dordrecht, 1988, pp. 113-125. 6. Kulkarni R. Proc. Roy. Soc. London Ser. A, 401, 117-130 (1985). 7. Ovsienko V. Yu., Tabachnikov S. Selecta Math. (N.S.), 2, No. 2, 297-307 (1996). 8. Tabachnikov S. In: Amer. Math. Soc. Transl., Ser. 2, vol. 180 (34), 1997, pp. 229-239. 9. Wolf J. A. Spaces of constant curvature. McGraw-Hill, New York, 1967.

Université de la Méditerranée and CPT-CNRS e-mail: duval@cpt.univ-mrs.fr

Поступило в редакцию 1 сентября 1998 г.

CNRS, Centre de Physique Théorique

e-mail: ovsienko@cpt.univ-mrs.fr

УДК $512.552 .8+512.6$

\section{Однопараметрические оснащенные частично упорядоченные множества и их представления}

\author{
(c) 2000. А. В. ЗАБАРИЛО, А. Г. ЗАВАДСКИЙ
}

Хорошо известно, что представления частично упорядоченных множеств были введены в начале 70-х годов Л. А. Назаровой и А. В. Ройтером [1] в связи с изучением представлений конечномерных алгебр [2]. Особенность матричных задач, соответствующих представлениям частично упорядоченных множеств, состоит в том, что в категории, определяющей такую задачу (и называемой вектроидом $\left.^{1}\right)$, все объекты и их кольца эндоморфизмов одномерны над заданным полем (см. [3]).

В настоящей работе эта классическая ситуация получает развитие в направлении рассмотрения таких матричных задач над полем вещественных чисел $\mathbb{R}$, которые соответствуют вектроидам не только с одномерными, но и с двумерными объектами, причем кольцо эндоморфизмов любого двумерного объекта предполагается изоморфным полю комплексных чисел $\mathbb{C}$. В этом случае вектроид задается частично упорядоченным множеством с некоторой простой дополнительной структурой, которое мы называем оснащенным. Цель работы дать полную классификацию представлений однопараметрических оснащенных частично упорядоченных множеств.

\footnotetext{
${ }^{1}$ Вектроид - это спектроид [3], вложенный в категорию векторных пространств.
} 
Перейдем к точным определениям и формулировке результатов.

Частично упорядоченное множество $\mathbf{P}$ назовем оснащенньлм, если выполняются три условия:

1) точки множества $\mathbf{P}$ делятся на одинарнье и двойнье;

2) отношения порядка между точками делятся на слабье и сильные;

3) если $x<y$ - слабое отношение, то точки $x, y$ двойные, и если при этом $x<t<y$, то отношения $x<t$ и $t<y$ тоже слабые (и автоматически точка $t$ двойная).

Условимся через $x \prec y(x \triangleleft y)$ обозначать слабое (сильное) отношение. Будем называть множество $\mathbf{P}$ обычнылм, если его оснащение тривиально (т. е. все точки являются одинарными).

Всякое оснащенное множество $\mathbf{P}$ определяет матричную задачу смешанного типа над парой $(\mathbb{R}, \mathbb{C})$. А именно, рассмотрим прямоугольную матрицу $M$, разделенную на вертикальные полосы $M_{x}(x \in \mathbf{P})$, где элементы полосы $M_{x}$ принадлежат полю $\mathbb{R}(\mathbb{C})$, если точка $x$ одинарная (двойная). Над матрицей $M$ разрешается осуществлять следующие преобразования:

(a) элементарные преобразования строк всей матрицы $M$ над $\mathbb{R}$;

(b) элементарные преобразования столбцов полосы $M_{x}$ над $\mathbb{R}$ (над $\mathbb{C}$ ), если точка $x$ одинарная (двойная);

(с) прибавления столбцов полосы $M_{x}$ к столбцам полосы $M_{y}$ с коэффициентами из $\mathbb{C}$, если отношение $x<y$ слабое;

(d) независимые прибавления действительных и мнимых частей столбцов полосы $M_{x}$ к действительным и мнимым частям (в любых сочетаниях) столбцов полосы $M_{y}$ с коэффициентами из $\mathbb{R}$, если отношение $x<y$ сильное.

Разделенные на полосы матрицы такого типа (называемые матричнылми представлениями множества $\mathbf{P}$ ) считаются эквивалентныли, если они переводятся друг в друга указанными преобразованиями, и задача заключается в классификации неразложимых в естественном смысле матриц с точностью до эквивалентности.

Ясно, что при тривиальном оснащении мы получим обычную задачу о представлениях частично упорядоченного множества в смысле [1] над полем $\mathbb{R}$. Если же множество $\mathbf{P}$ является антицепью (т. е. состоит из попарно несравнимых элементов), то возникает задача того же типа, что и в [4].

Размерность представления $M$ есть вектор $d=\underline{\operatorname{dim}} M=\left(d_{0} ; d_{x} \mid x \in \mathbf{P}\right)$, где $d_{0}$ - число строк матрицы $M$, a $d_{x}$ - число столбцов полосы $M_{x}$.

Если вектор $d$ не имеет нулевых координат, то его (и представление $M$ ) называют точнылм.

Представления оснащенного множества $\mathbf{P}$ над парой колец многочленов $(\mathbb{R}[t], \mathbb{C}[t])$ определяются аналогично случаю пары $(\mathbb{R}, \mathbb{C})$. Говорят, что множество бесконечного типа (т. е. имеющее бесконечно много неэквивалентных неразложимых представлений над парой $(\mathbb{R}, \mathbb{C}))$ является однопараметрическим, если существует его $(\mathbb{R}[t], \mathbb{C}[t])$-представление, которое порождает в стандартном смысле ${ }^{1}$ почти все неразложимые $(\mathbb{R}, \mathbb{C})$-представления любой фиксированной размерности.

Отметим, что представления обычных множеств конечного типа описаны М. М. Клейнером [6, 7], а однопараметрических — В. В. Отрашевской [8, 9].

${ }^{1}$ Путем подстановки вместо $t$ любой квадратной матрицы $A$ над $\mathbb{R}$, а вместо чисел $\lambda$ - скалярных матриц $\lambda E$ того же порядка. 
Полное решение вопроса о представлениях оснащенных множеств конечного типа следует из более общего результата Б. Клемп и Д. Симсона о шуровских вектроидах конечного типа [10].

Во всех упомянутых случаях получены списки точных множеств.

Каждому оснащенному множеству $\mathbf{P}$ сопоставляется квадратичная форма Tumca $f$ вида $f(d)=d_{0}^{2}+\sum_{x} f_{x} d_{x}^{2}+\sum_{x<y} p_{x y} f_{x} f_{y} d_{x} d_{y}-\sum_{x} f_{x} d_{x} d_{0}$, где $f_{x}=1$ $\left(f_{x}=2\right)$, если точка $x$ одинарная (двойная), и $p_{x y}=1\left(p_{x y}=1 / 2\right)$, если $x \triangleleft y$ $(x \prec y)$. Как обычно, корнями формы $f$ (множества $\mathbf{P}$ ) называются векторы, получаемые отражениями из простых корней (см. [5]), а мнимьлми корнями нули формы $f$ (ясно, что если $d$ - корень, то $f(d)=1$ или $f(d)=2$ ).

Назовем весом множества $\mathbf{P}$ наибольшую из сумм $\sum_{x \in X} f_{x}$, вычисленную для всевозможных антицепей $X \subset \mathbf{P}$.

Пусть $\left(m_{1}, \ldots, m_{n}\right)$ обозначает кардинальную сумму $n$ обычных цепей с числом точек $m_{1}, \ldots, m_{n}$ соответственно, а $(N, m)$ - кардинальную сумму (тоже неоснащенную) $m$-точечной цепи и множества $N=\{x<y>z<t\}$. Будем рассматривать также кардинальные суммы типа $\left(\widetilde{m}_{1}, \widetilde{m}_{2}\right),\left(\widetilde{m}_{1}, m_{2}\right),\left(\widetilde{m}_{1}, m_{2}, m_{3}\right)$, где $\widetilde{m}$ обозначает цепь из двойных точек $x_{1} \prec x_{2} \prec \cdots \prec x_{m}$, такую, что $x_{1} \prec$ $x_{m}$ при $m>1$. Множества вида $K_{1}=(1,1,1,1), K_{2}=(2,2,2), K_{3}=(1,3,3)$, $K_{4}=(N, 4), K_{5}=(1,2,5)$ и $M_{1}=(\tilde{1}, \tilde{1}), M_{2}=(\tilde{3}, 1), L_{1}=(\tilde{1}, 1,1), L_{2}=(\tilde{2}, 2)$ принято называть критическими $[6,10]$.

ТЕОРемА 1. Оснащенное частично упорядоченное множество является однопараметрическим тогда и только тогда, когда оно имеет вес $\leqslant 4$ и содержит ровно одно критическое подмножество.

Теорема 2. Для того чтобь вектор $d>0$, соответствуюший оснащенному однопараметрическому множеству, бъл размерностью неразложимого представления, необходимо и достаточно, чтобы он был корнем, мнимьлм корнем или особым вектором (особье векторы перечисленьл в замечании ниже). При этом

(а) если $d$ - корень или особьий вектор, то существует ровно одно (с точностью до изоморфизма) неразложимое представление размерности $d$;

(b) если $d$ - мнимьй корень, то существует бесконечно много неизомор фньх неразложимьх представлений размерности $d$.

Теорема 3. Нетривиально оснащенное однопараметрическое частично упорядоченное множество является точным тогда и только тогда, когда оно изоморфно или антиизоморфно одному из следующих 28 множеств (буквами а, $b, p, q$ обозначень двойнье точки, буквами $x, y, u, v$ - одинарнье): $M_{1}, M_{2}$, $M_{3}=\{a<x, b\}, M_{4}=\{a<x, b<y\}, M_{5}=\{a<x<y, b\}, M_{6}=\{x<a, b<y\}$, $M_{7}=\{x<a<y, b\}, M_{8}=\{a \prec p \succ b\}, M_{9}=\{x>a \prec p \succ b\}, M_{10}=\{p \prec(a, b) \prec q$, где $p \prec q\}, M_{11}=\{(a, b) \prec p \prec q$, где $(a, b) \prec q\}, M_{12}=\{a>x<y>b\}, M_{13}=$ $\{p \prec(a, b) \prec q$, где $p \triangleleft q\}, L_{1}, L_{2}, L_{3}=\{a<x, u, v\}, L_{4}=\{a, u<x>v\}, L_{5}=$ $\{a<x<y, u, v\}, L_{6}=\{a<x,(u, v)<y\}, L_{7}=\{a,(u, v)<x<y\}, L_{8}=\{a<x$, $y<(u, v)\}, L_{9}=\{x<a<y, u, v\}, L_{10}=\{a, x<(u, v)<y\}, L_{11}=\{a \prec p>u, v\}$, $L_{12}=\{p \prec a \prec q>u>p, v\}, L_{13}=\{u>p \prec a \prec q>v$, где $p \prec q\}, L_{14}=\{a>x<y$ $>(u, v)\}, L_{15}=\{u>p \prec a \prec q>v$, zде $p \triangleleft q\}$.

[Здесь $(x, y)<z$ означает $x<z$ и $y<z$ (аналогично обозначение используется для символов $>, \prec, \succ)$.] 
ЗАмечАниЕ. Точный вектор $d$, соответствующий нетривиально оснащенному однопараметрическому множеству $\mathbf{P}$, является особълм тогда и только тогда, когда множество $\mathbf{P}$ (рассматриваемое с точностью до антиизоморфизма) совпадает с одним из множеств $M_{12}, M_{13}, L_{14}, L_{15}$ и $d=\left(d_{0} ; d_{a}, d_{b}, d_{x}, d_{y}\right)=$ $(2 k+1 ; k, k, 1,1)$ при $\mathbf{P}=M_{12}, d=\left(d_{0} ; d_{a}, d_{b}, d_{p}, d_{q}\right)=(2 k+2 ; k, k, 1,1)$ при $\mathbf{P}=M_{13}, d=\left(d_{0} ; d_{a}, d_{u}, d_{v}, d_{x}, d_{y}\right)=(2 k+1 ; k, k, k, 1,1)$ при $\mathbf{P}=L_{14}, d=$ $\left(d_{0} ; d_{a}, d_{u}, d_{v}, d_{p}, d_{q}\right)=(4 k+2 ; 2 k, 2 k, 2 k, 1,1)$ при $\mathbf{P}=L_{15}(k \geqslant 1)$. При этом $f(d)=2$ в случае $\mathbf{P}=M_{12}, L_{14}$ и $f(d)=4$ в случае $\mathbf{P}=M_{13}, L_{15}$ (об особых векторах обычных однопараметрических множеств см., например, [11]).

В ходе работы получен также полный список неразложимых представлений всех перечисленных множеств (и им антиизоморфных) в матричной форме.

Доказательство основано на разработке и применении алгоритмов дифференцирования оснащенных множеств (развивающих алгоритмы, описанные в $[1,11])$, использовании результатов работы [12] и непосредственных вычислениях.

Авторы благодарны рецензенту за полезные замечания.

\title{
ЛИтеРАтУРА
}

1. Назарова Л. А., Ройтер А. В. Зап. научн. сем. ЛОМИ, 28, 5-31 (1972). 2. Назарова Л. A., Ройтер А. В. Препринт 73.9 ИМ АН УССР (1973). 3. Gabriel P., Roiter A. $V$. Representations of finite-dimensional algebras. Algebra VIII, Encycl. Math. Sci., vol. 73, Springer-Verlag, 1992. 4. Dlab V., Ringel C. M. Indecomposable representations of graphs and algebras. Mem. Amer. Math. Soc., 6, No. 173 (1976). 5. Poümep A. B. Труды МИАН, 148, 201-210 (1978). 6. Клейнер М. М. Зап. научн. сем. ЛОМИ, 28, 32-41 (1972). 7. Клейнер М. М. Зап. научн. сем. ЛОМИ, 28, 42-59 (1972). 8. Отрашевская В. В. Укр. матем. ж., 28, № 3, 334-341 (1976). 9. Отрашевская В. В. В сб.: Матричные задачи, Киев, 1977, с. 144-149. 10. Klemp B., Simson D. J. Algebra, 134, No. 2, 390-468 (1990). 11. Завадский А. Г. Изв. АН СССР, сер. матем., 55, № 5, 10071048 (1991). 12. Dlab V., Ringel C. M. Linear Algebra Appl., 17, 107-124 (1977).

Киевский национальный университет

строительства и архитектуры

Поступило в редакцию 30 декабря 1997 г.

УДК $515.16+517.9$

\section{Слабые ККС и КАС и индуктивные пределы семейств групп и алгебр*}

\author{
(c) 2000. Р. С. ИСМАГИЛов
}

1. Еще о канонических коммутационных и антикоммутационных соотношениях (кратко $\mathrm{KKC}$ и $\mathrm{KAC}$ ). Пусть $V$ - вещественное линейное пространство и $\tau$ - невырожденная кососимметрическая форма на $V$. Тогда $\mathrm{KKC} \mathrm{-} \mathrm{это} \mathrm{семейство} \mathrm{унитарных} \mathrm{операторов}\{U(x), x \in V\}$ (действующих в некотором гильбертовом пространстве), таких, что

$$
U(x+y)=\exp (i \tau(x, y)) U(x) U(y) \quad(x, y \in V)
$$

* Исследование поддержано РФФИ, грант № 96-15-96249. 